\title{
Psychological and Physiological Profiles in Oncology Caregivers: a Multivariable Cross-Sectional Study
}

\author{
Esther García ${ }^{a}$, Sira Aguilób ${ }^{b}$ Adriana Arza ${ }^{a, c}$, Jorge M. Garzón-Reyac, Jordi Aguilóa,c \\ ${ }^{a}$ CIBER-BBN, Barcelona, Spain, ${ }^{b}$ Hospital Clínic, Barcelona, Spain, ${ }^{c}$ Universitat Autònoma de Barcelona, \\ Barcelona, Spain \\ acmlis.conference@gmail.com
}

\begin{abstract}
Scientific research has led to enhanced medical assistance, and hence higher disease control and a continuous lifespan dilation. However, research mainly focusses on the patient's condition, and, in some cases, caregivers' health is undervalued. In this paper, a close-related emotionally involved caregiver group is examined and compared to a non-caregiver group in order to investigate the role of chronic stress in their psychological and physiological state. For this purpose, blood, saliva, and hair samples, physiological signals and three validated psychometric tests are collected from both groups. Results from 39 subjects show interesting significant differences among all the psychometric profiles between the two groups although no sufficient biochemical data supports the relation with potential health consequences.

Keywords: Oncology caregivers; Stress biomarkers; Chronic stress; Psicometric tests

\section{Introduction}

Scientific research has led to enhanced medical assistance, and hence higher disease control and a continuous lifespan dilation. However, research mainly focusses on the patient's condition, and, in some cases, caregivers' health is undervalued. Informal caregivers are non-remunerated and non-professional people in charge of the care of a family member with limited autonomy due to physical or cognitive impairment.

Daily caregiving leads to a burden escalation with physical and psychological impact on the caregiver health $[1,2]$. The unrelenting repercussions on caregivers' health come from the physical burden related to the daily tasks, the psychological frustration after the inevitable deterioration of the cherished patient and even the funding of the care expenses can passively impact a person's wellbeing $[3,4]$ Common symptoms are sleeping disturbance, irritability, fatigue, weight gain or loss without diet, frequent colds and infections among others [3,5]. From a psychological point of view, the subjective reaction towards the care receptor demands often lead to psychological problems such as depression, anxiety and stress, collectively referred to as the caregiver stress syndrome $[3,5,6]$. Some studies have shown that stress is an independent risk factor of acute myocardial infarction, comparable to arterial hypertension and abdominal obesity, diabetes mellitus type 2, psychiatric diseases and implication in the development of Alzheimer's disease [7-10].
\end{abstract}


Esther García, Sira Aguiló, Adriana Arzaa, Jorge M. Garzón-Reya, Jordi Aguiló. Psychological and Physiological Profiles in Oncology Caregivers: a Multivariable Cross-Sectional Study. Transactions on Machine Learning and Artificial Intelligence, Vol 5 No4 August (2017); p: 547-557

Stress factors induce homeostasis disturbance in the organism and the coping mechanisms to recuperate the equilibrium are known to be mainly regulated through Autonomic Nervous System and the Hypothalamus-Pituitary-Adrenal axis (HPAA) [11]. These adaptations include endocrine, electrophysiological and/or psychological changes that become pathologic if they are not limited to an adaptive and short-in-time response.

The fastest response noticeable are the electrophysiological signs coming from the activation of the autonomic nervous system. The information of the balance between the activation of the sympathetic and parasympathetic nervous systems can be provided by a wide variety of measures; for instance, the heart rate and its components: heart rate variability (HRV) low and high frequency ratios (LF/HF) and R to $R$ peaks variability (RRV) [12-14]. There are also reports of an increase in the respiratory rate (RR) and its variability (RRV) in front of a stressor [15]. Other studies have established a relationship between stress and blood pressure (BP), the sweat-dependent electrical characteristics of the skin (GSR - galvanic skin response) and the skin temperature (ST) [16-19].

A parallel slower response is mediated through the HPAA initiated in the paraventricular nucleus of the hypothalamus. This endocrine response can be revealed by means of several biomarkers. For instance, reported data show quick variations in blood glucocorticoid levels (cortisol in humans) due to the action of stressors [20]. However, salivary and hair samples seem to be growing non-invasive and more stable measures of cortisol concentrations [21,22]. Similarly, $\alpha$-amylase is known to be a marker of the activity of the sympathetic nervous system in response to stress. Copeptin, a surrogate biomarker of vasopressin (AVP), is another possible marker of stress, and it is quite stable both in circulation and ex-vivo. Levels of copeptin are sensitive to situations involving physical stress [23], but could also be sensitive to emotional stress [24]. Inflammation biomarkers such as interleukin 6 and 10 (IL6, IL10) and tumor necrosis factor alpha (TNF- $\alpha$ ) have been associated with depressive disorders as well [25].

Considering the psychological branch, psychometric tests are the most commonly accepted tools for measuring emotional distress. The PSS, STAI and VASS are validated, endorsed and very well documented by the medical world [26-28]. They are the reference standard for helping to determine the level of stress reached by the subject in each situation.

In spite of the wide variety of studies looking for a unique parameter to measure stress, there are several throwbacks. For instance, the multidimensional nature of the questioners and the lack of standardization hinder the identification of the most appropriate test to measure objectively the stress level [29]. Additionally, since the HPA axis is a highly adaptive system which is characterized by marked inter- and intraindividual variability, the development of such markers of HPA axis regulation in humans is still a rather challenging task.

Bearing all this information in mind, and considering that nowadays, there is still not an objective measure of signs that allow the early diagnosis of stress, ES3 project aims to find a multivariable approach, quantitatively and objectively assessing the three branches affected, that could provide very valuable information to detect and prevent symptoms and diseases associated with the acute and chronic stress [30]. 
The present study shows some preliminary results of a pilot included in the bigger project mentioned above [30]. This pilot is focused in chronic stress in oncology caregivers, evaluating biochemical, electrophysiological and psychometric data. According to the World Health Organization, cancer is the second leading cause of death globally, and for many oncology patients, palliative care is the only available treatment usually involving highly demanding home-based care. Early diagnosis of the caregiver's stress syndrome along with home care programs and nursing support could prevent chronic stress effects and reduce the development of stress-related diseases in this population.

The aim of this work is to assess the perceived stress and endocrine, metabolic and immunologic profile comparing an oncology informal caregivers group with a matched comparison group.

\section{Patients and Methods}

\subsection{Patients}

Caregivers of oncologic patients (oncology caregivers) attending at the Oncology Service of the Hospital Clínic of Barcelona (HCPB, Barcelona, Spain), were invited to participate in the cross-sectional study. A paired group of non-caregivers volunteers unrelated with the patients were invited to participate in this study.

The study was performed in the Hospital Clínic of Barcelona between September 2015 and June 2016. The study was approved by the institutional Committee of Ethics and in accordance with those of the World Medical Association and the Helsinki Declaration. All participants were informed about the purpose and characteristics of the study and their rights to withdraw of the study at any time during process. All participants signed the informed consent.

Inclusion criteria for caregivers were: > 18 years old, to take care of an oncological patient with progressive incurable cancer and oncospecific treatment, to be unpaid for the caregiving tasks, to live with the patient in the same household, to be emotionally involved (family or partner), and to develop the caregiver activity for a minimum period of 6 months.

Inclusion criteria for non-caregivers were: to be at least 18 years old and not to be in charge of any family requiring care. Non-caregivers were matched for age (up to 5 years older or younger), gender and body mass index (BMI).

Exclusion criteria for all participants were any of: failure to meet any of the inclusion criteria; habitual intake of psychotropic or corticoid; to be diagnosed with alteration of the hypothalamic axis adrenal pituitary; or to have a Body Mass Index (BMI) greater than 35.

\subsection{Endpoints and Methods}

Socio-demographic and lifestyle variables analysed were: age, gender, BMI, habits (smoking status, coffee and alcohol intake, consume of drugs and physical activity), health condition (chronic disease diagnosed, medication and psychotropic treatment) and other variables (cohabitation condition and relationship and job status). Detailed characteristics of these variables are shown in Table 1.

Primary endpoints were perceived stress scores using Spanish version of psychometric tests: Perceived Stress Scale (PSS) [26], State-Trait Anxiety Inventory (STAI) [28], and Stress Visual Analogue Scale (VASS) [27]. 
Esther García, Sira Aguiló, Adriana Arzaa, Jorge M. Garzón-Reya, Jordi Aguiló. Psychological and Physiological Profiles in Oncology Caregivers: a Multivariable Cross-Sectional Study. Transactions on Machine Learning and Artificial Intelligence, Vol 5 No4 August (2017); p: 547-557

PSS provides a global measure of perceived stress in a situation appraised as stressful and considering the feelings and thoughts occurred during the last month. The test consists in a 10 questions survey with liker scaling responses (never, almost never, sometimes, fairly often and very often). STAI measures a subjective emotional state. The test consists of 40 total questions with liker scaling responses, 20 out of 40 total questions measure the trait anxiety (almost never, sometimes, often and almost always) and the remaining 20 out of 40 total questions measure the state anxiety (not at all, somewhat, moderately so, very much so). VASS consists in a simple visual 100-point scale (0, not at all; 100, absolutely stressed), in which participants rate their present perceived stress. Tests take few minutes for the patient to complete and no special education or training is required to administer them.

Secondary endpoints were endocrine, metabolic and inflammatory markers: plasma copeptin (pmol/L), copeptin osmolarity (mOsm), glucose (mg/dL), glycated hemoglobin (HbA1c; IFCC and NGSP/DCCT units) (\%), LDL (mg/dL), HDL (mg/dL), cholesterol $(\mathrm{mg} / \mathrm{dL})$, triglycerides $(\mathrm{mg} / \mathrm{dL})$, salivary $\alpha$-amylase $(\mathrm{U} / \mathrm{mL})$, salivary cortisol $(\mu \mathrm{g} / \mathrm{dL})$, hair-cortisol $(\mathrm{pg} / \mathrm{mg}), \mathrm{TNF} \alpha(\mathrm{pg} / \mathrm{mL}), \mathrm{IL}-6(\mathrm{pg} / \mathrm{mL})$ and IL-10 $(\mathrm{pg} / \mathrm{mL})$. These analytes can result in stress biomarkers and information of the overall health of the subjects.

Plasma samples were obtained from $10 \mathrm{~mL}$ of blood collected in serum tubes for the biochemical and immunological analysis and ethylenediaminetetraacetic acid (EDTA) tubes for the HbA1c and copeptin analysis. Salivary samples were collected with salivettes (Sarstedt, Granollers, Spain) and stored at -20ㄷ until the analysis day. Then, salivettes were thawed at room temperature and centrifuged at 4 으 for 10 minutes at 1000g. Salivary $\alpha$-amylase was analysed using Salimetrics Salivary Alpha-Amylase Assay

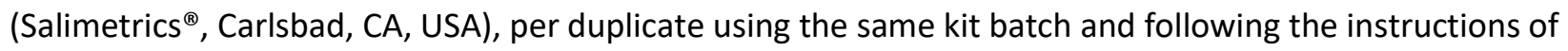
the manufacturer. The amount of $\alpha$-amylase activity is directly proportional to the $405 \mathrm{~nm}$ absorbance. Salivary cortisol was analysed using Salimetrics Cortisol Enzyme Immunoassay (Salimetrics ${ }^{\circledR}$, Carlsbad, CA, USA), by duplicate using the same corresponding kit batch and following the instructions of the manufacturer. The limit of quantification of cortisol assay is $0.012 \mu \mathrm{g} / \mathrm{dL}$.

Hair samples were stored at room temperature in propylene tubes protected from light. The accumulated cortisol was extracted according to the procedure by Scorrano et al. with minor modifications [31]. Briefly, the $3 \mathrm{~cm}$ of hair closest to the scalp were obtained and washed two times for 1 minute in $10 \mathrm{ml}$ of isopropanol and completely air-dried at room temperature. For the extraction of cortisol, $40 \mathrm{mg}$ of hair was mixed with $1.6 \mathrm{~mL}$ of methanol overnight by continuous rotation ( $20 \mathrm{rpm} / \mathrm{min})$. After centrifugation $\left(10,000 \mathrm{~g}, 10 \mathrm{~min}, 4^{\circ} \mathrm{C}\right)$, the methanol was recovered in a new clean glass tube and the procedure was repeated again. Total recovered methanol was pooled and dried up under a nitrogen stream. Cortisol extracts were reconstituted in $200 \mu \mathrm{L}$ of sodium phosphate buffer and analysed by using Salimetrics ${ }^{\circledR}$ Cortisol Enzyme Immunoassay. The template is used to format your paper and style the text. All not revise any of the current designations.

All the biochemical analysis were processed at the Biomedical Diagnosis Centre at the Hospital Clínic of Barcelona. Cortisol and $\alpha$-amylase in saliva and hair samples were processed in the laboratory of the Endocrinology and Radioimmunoanalysis Service of the Animal Physiology Unit at the Universitat Autònoma de Barcelona, and TNF $\alpha$, IL-6 and IL-10 were sent to the Myeloid Cell Laboratory at the Biological Research Centre in Madrid. 


\subsection{Data analysis}

Descriptive analysis of data was performed with $\mathrm{SAS}^{\circledR}$ software version 9.4 and Matlab R2014b. For categorical variables, percentages of events were calculated and the comparisons of groups were analysed by Fisher's exact test. For quantitative variables, the median and the inter-quartile range (IQR) were calculated. Comparisons among groups were analysed by non-parametric analysis (Wilcoxon Signed Rank Test) according to the sample size and criteria of normality (Shapiro-Wilk test). All tests were twotailed, and p-values $<0.05$ were considered statistically significant.

\section{Results}

A total of 39 subjects participated in the study (19 non-caregivers and 20 oncology caregivers). The median age (IQR) of participants was 55 years (45-64), the median (IQR) of BMI was 25.56 (21.60-28.39) and there was a majority gender group with $66.67 \%$ of women. Non-significant differences were found among groups in terms of gender, age and BMI (Fisher's Exact and Wilcoxon signed rank tests $p$-value $>0.05$ ) (Table I).

Table I. Sociodemographic and lifestyle characteristics.

\begin{tabular}{|c|c|c|c|}
\hline & Non-Caregivers & Oncologic Caregivers & Total \\
\hline Participants & $19(48.72)$ & $20(51.28)$ & $39(100.00)$ \\
\hline Age. yr & $\begin{array}{c}53.00 \\
(45.00-65.00)\end{array}$ & $\begin{array}{c}55.50 \\
(47.00-62.00)\end{array}$ & $\begin{array}{c}55.00 \\
(45.00- \\
64.00)\end{array}$ \\
\hline \multicolumn{4}{|c|}{ Sex } \\
\hline Men & $6(31.58)$ & $7(35.00)$ & $13(33.33)$ \\
\hline Women & $13(68.42)$ & $13(65.00)$ & $26(66.67)$ \\
\hline$B M I$ & $\begin{array}{c}25.61 \\
(20.83-28.39)\end{array}$ & $\begin{array}{c}25.40 \\
(22.24-28.04)\end{array}$ & $\begin{array}{c}25.56 \\
(21.60- \\
28.39)\end{array}$ \\
\hline \multicolumn{4}{|c|}{ Chronic disease } \\
\hline Non & $15(78.95)$ & $8(40.00)^{*}$ & $23(58.97)$ \\
\hline Yes & $4(21.05)$ & $12(60.00)^{*}$ & $16(41.03)$ \\
\hline \multicolumn{4}{|c|}{ Medication } \\
\hline Non & $11(57.89)$ & $9(45.00)$ & $20(51.28)$ \\
\hline Yes & $8(42.11)$ & $11(55.00)$ & $19(48.72)$ \\
\hline \multicolumn{4}{|c|}{ Psychotropic } \\
\hline Non & $17(89.47)$ & $14(70.00)$ & 31 (79.49) \\
\hline Occasionally & $2(10.53)$ & $6(30.00)$ & $8(20.51)$ \\
\hline \multicolumn{4}{|c|}{ Smoker } \\
\hline Non & $18(94.74)$ & $12(60.00)^{*}$ & $30(76.92)$ \\
\hline Yes & $1(5.26)$ & $8(40.00)^{*}$ & $9(23.08)$ \\
\hline \multicolumn{4}{|c|}{ Daily Coffee } \\
\hline None & $1(5.26)$ & $1(5.00)$ & $2(5.13)$ \\
\hline$\geq 1$ cup & $18(94.74)$ & $19(95.00)$ & $37(94.87)$ \\
\hline \multicolumn{4}{|c|}{ Daily Alcohol } \\
\hline None & $2(10.53)$ & $9(45.00)^{*}$ & $11(28.21)$ \\
\hline$\geq 1$ glass & $17(89.47)$ & $11(55.00)^{*}$ & $28(71.79)$ \\
\hline \multicolumn{4}{|c|}{ Sport practice } \\
\hline Non & $3(15.79)$ & $15(75.00)$ & $18(46.15)$ \\
\hline (2) Yes & $16(84.21)$ & $5(25.00)$ & $21(53.85)$ \\
\hline
\end{tabular}


Esther García, Sira Aguiló, Adriana Arzaa, Jorge M. Garzón-Reya, Jordi Aguiló. Psychological and Physiological Profiles in Oncology Caregivers: a Multivariable Cross-Sectional Study. Transactions on Machine Learning and Artificial Intelligence, Vol 5 No4 August (2017); p: 547-557

\begin{tabular}{|l|c|c|c|}
\hline \multicolumn{5}{|c|}{ Married } \\
\hline Non & $8(42.11)$ & $5(25.00)$ & $13(33.33)$ \\
\hline Yes & $11(57.89)$ & $15(75.00)$ & $26(66.67)$ \\
\hline \multicolumn{4}{|c|}{ Cohabitation } \\
\hline Family & $6(31.58)$ & $8(40.00)$ & $14(35.90)$ \\
\hline Partner & $8(42.11)$ & $9(45.00)$ & $17(43.59)$ \\
\hline Alone & $5(26.32)$ & $3(15.00)$ & $8(20.51)$ \\
\hline \multicolumn{4}{|c|}{ Job status } \\
\hline Inactive & $4(21.05)$ & $10(50.00)$ & $14(35.90)$ \\
\hline Active & $15(78.95)$ & $10(50.00)$ & $25(64.10)$ \\
\hline
\end{tabular}

(1) Less than twice/week and any during the last 24hrs. (2)Either occasionally or currently. Abbreviations: yr, years. (*) Group comparison statistically significant ( $p$-value $<0.05$ ). Fisher's exact test for discrete variables, $N(\%)$ and Wilcoxon Signed Rank Test for quantitative variables, Median (IQR).

Even though the two groups showed similar medication and psychotropic consumption (Fisher's Exact Test, $p=0.1842$ and $p=0.2351$, respectively), the oncology caregivers' group manifested a higher chronic disease incidence of $60 \%$ compared to the $21.05 \%$ of the non-caregiver group (Fisher's Exact Test, $p=$ 0.0225). Both groups presented similar social relations, with similar cohabitation, relationship and job status (Fisher's Exact Test, $p$-values $>0.05$ ). More than 1 cup of coffee per day was the usual intake of the $95 \%$ (37/39) of total participants (Fisher's Exact Test, $p=1.0000)$. A 40\% (8/12) of caregiver subjects were smokers, a significantly greater proportion than in the comparison group where there was only 1 out of 19 (5.26\%, Fisher's Exact Test, $p=0.0197)$. On the contrary, the highest percentage in daily alcohol intake was in the non-caregivers group (89.47\%, Fisher's Exact Test, $p=0.0310)$. A $53.85 \%$ (21/39) of total participants practiced sport. However, the percentage of physically active subjects was considerably bigger in the comparison group than in the caregiver's group ( $84.21 \%$ and $25 \%$ respectively, Fisher's Exact Test, $\mathrm{p}=0.0003$ ) (Table I).

Table 2. Psychometric tests scores

\begin{tabular}{|c|c|c|c|}
\hline & Non-caregivers & Oncologic caregivers & \multirow{3}{*}{$\begin{array}{c}\text { Wilcoxon } \\
\text { Signed Rank } \\
\text { Test }\end{array}$} \\
\hline & Median (IQR) & Median (IQR) & \\
\hline & $N=19$ & $N=20$ & \\
\hline PSS & $\begin{array}{c}22.00 \\
(18.00-23.00)\end{array}$ & $\begin{array}{c}24.50 \\
(23.00-27.50)\end{array}$ & $\begin{array}{c}S=75.5 \\
p=0.0012^{*}\end{array}$ \\
\hline STAI-S & $\begin{array}{c}11.00 \\
(6.00-16.00)\end{array}$ & $\begin{array}{c}47.50 \\
(35.00-53.00)\end{array}$ & $\begin{array}{c}S=95 \\
p<0.0001^{*}\end{array}$ \\
\hline STAI-t & $\begin{array}{c}13.00 \\
(9.00-21.00)\end{array}$ & $\begin{array}{c}28.50 \\
(20.00-40.00)\end{array}$ & $\begin{array}{c}S=80.5 \\
p=0.0004^{*}\end{array}$ \\
\hline VASS & $\begin{array}{c}40.00 \\
(23.00-50.00)\end{array}$ & $\begin{array}{c}80.00 \\
(65.00-82.50)\end{array}$ & $\begin{array}{c}S=95 \\
p<0.0001^{*}\end{array}$ \\
\hline $\begin{array}{l}\text { (2)Summary } \\
\text { score }\end{array}$ & $\begin{array}{c}99.00 \\
(67.00-116.00)\end{array}$ & $\begin{array}{c}218.50 \\
(190.00-249.00)\end{array}$ & $\begin{array}{c}S=94 \\
p<0.0001^{*}\end{array}$ \\
\hline
\end{tabular}

(1) Summary score obtained from the sum of psychometric test scores of each participant for a general overall analysis. (*) Group comparison statistically significant ( $p$-value $<0.001$ ).

Perceived stress scores analysed showed significantly different self-perceived stress profiles in all the psychometric tests given. We found evidences that the level of perceived stress in oncology caregivers is higher than the non-caregivers (Wilcoxon signed rank tests $p$-value <0.001). Oncology caregivers presented the highest score distribution in all psychometric tests: PSS test (median: 24.50; IQR: 23.0027.50), STAI-s test (median: 47.50; IQR: 35.00-53.00), STAI-t test (median: 28.50; IQR: 20.00-40.00) and in VASS rate (median: 80.00; IQR: 65.00-82.50) (Table II). The differences between the scores in both groups 
were the more noticeable in the STAI-s test and the VAS scale. The summary score is obtained from the sum of psychometric test scores of each participant for a general overall analysis and this combination shows the most pronounced difference (Fig. 1).

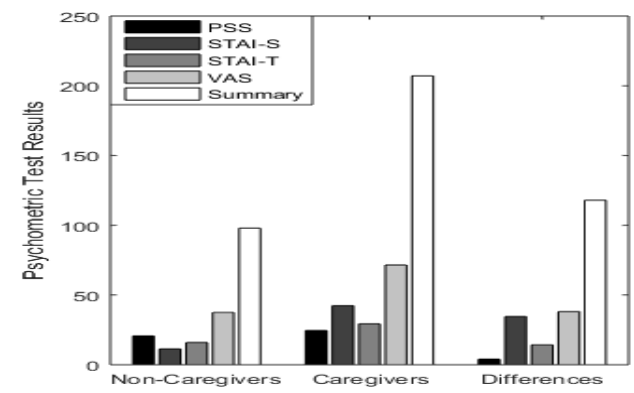

Figure 1. Psychometric test results from both the oncology caregivers and comparison groups and the score differences

Table 3. Endocrine, metabolic and inflammatory analytes concentrations.

\begin{tabular}{|c|c|c|c|}
\hline & $\begin{array}{c}\text { Non-caregivers } \\
\text { Median (IQR) }\end{array}$ & $\begin{array}{c}\text { Caregivers } \\
\text { Median (IQR) }\end{array}$ & $\begin{array}{c}\text { Wilcoxon } \\
\text { Signed } \\
\text { Rank Test }\end{array}$ \\
\hline Copeptin (pmol/L) & $\begin{array}{c}5.45 \\
(2.25-11.50)\end{array}$ & $\begin{array}{c}6.20 \\
(3.50-8.60)\end{array}$ & $\begin{array}{l}S=15.5 \\
p=0.5226\end{array}$ \\
\hline $\begin{array}{l}\text { Copeptin Osmolarity } \\
\text { (mOsm); }\end{array}$ & $\begin{array}{c}300.00 \\
(296.00-304.50)\end{array}$ & $\begin{array}{c}303.00 \\
(298.00-307.00)\end{array}$ & $\begin{array}{c}S=43 \\
p=0.0614\end{array}$ \\
\hline Glucose (mg/dL) & $\begin{array}{c}93.00 \\
(89.00-101.00)\end{array}$ & $\begin{array}{c}101.00 \\
(90.00-132.00)\end{array}$ & $\begin{array}{l}S=41.5 \\
p=0.0976\end{array}$ \\
\hline HbA1c (IFCC) (\%) & $\begin{array}{c}5.00 \\
(4.90-5.20)\end{array}$ & $\begin{array}{c}5.20 \\
(4.70-5.60)\end{array}$ & $\begin{array}{c}S=20 \\
p=0.3992\end{array}$ \\
\hline $\begin{array}{c}H b A 1 c \text { (NGSP/DCCT) } \\
\text { (\%) }\end{array}$ & $\begin{array}{c}5.40 \\
(5.30-5.60)\end{array}$ & $\begin{array}{c}5.60 \\
(5.10-6.00)\end{array}$ & $\begin{array}{c}S=20 \\
p=0.3992\end{array}$ \\
\hline$L D L(m g / d L)$ & $\begin{array}{c}109.70 \\
(100.00-135.40)\end{array}$ & $\begin{array}{c}127.60 \\
(104.60-154.00)\end{array}$ & $\begin{array}{l}S=-22.5 \\
p=0.3465\end{array}$ \\
\hline$H D L(m g / d L)$ & $\begin{array}{c}58.00 \\
(51.00-67.00)\end{array}$ & $\begin{array}{c}58.00 \\
(53.00-71.00)\end{array}$ & $\begin{array}{l}S=-31.5 \\
p=0.1811\end{array}$ \\
\hline Cholesterol ( $\mathrm{mg} / \mathrm{dL})$ & $\begin{array}{c}199.00 \\
(172.00-214.00)\end{array}$ & $\begin{array}{c}208.00 \\
(181.00-244.00)\end{array}$ & $\begin{array}{c}S=8.5 \\
p=0.7454\end{array}$ \\
\hline Triglycerides ( $\mathrm{mg} / \mathrm{dL}$ ) & $\begin{array}{c}81.00 \\
(58.00-96.00)\end{array}$ & $\begin{array}{c}77.00 \\
(57.00-156.00)\end{array}$ & $\begin{array}{c}S=15 \\
p=0.5678\end{array}$ \\
\hline $\begin{array}{c}\text { Salivary } \alpha \text {-amylase } \\
(U / \mathrm{mL})\end{array}$ & $\begin{array}{c}198.65 \\
(98.15-217.25)\end{array}$ & $\begin{array}{c}260.78 \\
(135.90-382.05)\end{array}$ & $\begin{array}{c}S=49 ; \\
p=0.0494^{*}\end{array}$ \\
\hline $\begin{array}{c}\text { Salivary cortisol } \\
(\mu g / d L)\end{array}$ & $\begin{array}{c}0.16 \\
(0.12-0.21)\end{array}$ & $\begin{array}{c}0.14 \\
(0.08-0.21)\end{array}$ & $\begin{array}{c}S=7 \\
p=0.7983\end{array}$ \\
\hline Hair-cortisol (pg/mg) & $\begin{array}{c}11.52 \\
(7.76-16.51)\end{array}$ & $\begin{array}{c}9.46 \\
(6.70-18.88)\end{array}$ & $\begin{array}{c}S=-8 ; \\
p=0.7680\end{array}$ \\
\hline$T N F a(p g / m L)$ & $\begin{array}{c}0.73 \\
(0.00-1.56)\end{array}$ & $\begin{array}{c}0.42 \\
(0.00-1.56)\end{array}$ & $\begin{array}{l}S=14.5 \\
p=0.4288\end{array}$ \\
\hline$I L-6(p g / m L)$ & $\begin{array}{c}1.15 \\
(0.00-3.46)\end{array}$ & $\begin{array}{c}2.40 \\
(0.00-5.38)\end{array}$ & $\begin{array}{c}S=11.5 \\
p=0.5016\end{array}$ \\
\hline$I L-10(p g / m L)$ & $\begin{array}{c}3.00 \\
(2.27-3.74)\end{array}$ & $\begin{array}{c}3.15 \\
(1.53-3.74)\end{array}$ & $\begin{array}{l}S=-23.5 \\
p=0.2787\end{array}$ \\
\hline
\end{tabular}

$\left.{ }^{*}\right)$ Group comparison statistically significant (p-value $\left.<0.05\right)$.

With respect to the biochemical analytes studied, the only levels that significantly differed were the $\alpha$ amylase concentrations (Wilcoxon Signed Rank Test, $p$-value $=0.0494$ ). Distribution values of concentration of glucose, glycated hemoglobin, copeptin, LDL, HDL, cholesterol, triglycerides, salivary 
Esther García, Sira Aguiló, Adriana Arzaa, Jorge M. Garzón-Reya, Jordi Aguiló. Psychological and Physiological Profiles in Oncology Caregivers: a Multivariable Cross-Sectional Study. Transactions on Machine Learning and Artificial Intelligence, Vol 5 No4 August (2017); p: 547-557

cortisol, hair-cortisol, TNF $\alpha$, IL- 6 and IL-10 did not differ statistically significant between non-caregivers and oncology caregivers groups (Wilcoxon Signed Rank Test, p-value >0.05) (Table III). Several clinically relevant concentrations were found in both groups although mainly in the caregivers' group (normal values of the CDB: glucose $65-110 \mathrm{mg} / \mathrm{dL}, \mathrm{HbA1c}<6.5 \%$ IFCC, LDL $<180 \mathrm{mg} / \mathrm{dL}, \mathrm{HDL}>40 \mathrm{mg} / \mathrm{dL}$, total cholesterol $148-247 \mathrm{mg} / \mathrm{dL}$, triglycerides $50-150 \mathrm{mg} / \mathrm{dL}$ ). We observed abnormal concentrations of glucose typed as pre-diabetes in some oncology caregivers.

\section{Discussion and conclusions}

The present study compares the self-perceived stress and endocrine, metabolic, and inflammatory analytes concentrations in an oncology caregiver group and a non-caregivers group. Our results provide consistent evidences that subjects with caregiver burden perceived much higher stress.

Cancer diagnosis and follow-up brings an important stress impact in cancer patients and relatives. Regular clinical visits, aggressive drug-treatments and health deterioration become more evident over time. These circumstances together with the unexpected care tasks of a family member are the root of great emotional and physical burden. PSS as well as STAI-t give a global measure of perceived stress and anxiety in front of a challenging situation considering the feelings and thoughts. The significant differences in both psychometric tests obtained reflect the tendency of this community to be more afflicted in front of situations appraised as stressful. Greater differences were observed among STAI-s and VAS scores which evaluate the stress felt at that precise moment. However, these results might be influenced by the impact of the setting for visit on oncology caregivers since they attended the study at the hospital during cancer patient visit.

Considering the analytes concentrations, our study assessed the levels of copeptin, glucose, glycated hemoglobin, LDL, HDL, cholesterol, triglycerides, $\alpha$-amylase, cortisol, TNF $\alpha$, IL- 6 and IL-10 in oncology caregivers and non-caregivers in a unique visit. Several studies report that psychological stressors may have physiological impact in the organism [1,21,23,24]. However, almost none of the endocrine, metabolic or immunologic biomarkers selected differed significantly between the two groups in our study. Salivary $\alpha$-amylase was the only analyte that showed statistically significant results. Similarly, Siddiqui A. et al. [10] found elevated levels of salivary $\alpha$-amylase as well as salivary cortisol in type 2 diabetes mellitus patients as stress responses. In our study, salivary cortisol levels were within the normal range. This discrepancy of the cortisol concentrations might be biased due to the diversity of stressors, the wide repertoire of mental stress measures, the sampling strategy and the intra-individual variability of diurnal cortisol fluctuation $[20,21]$. Other approaches suggest hair sampling to estimate cortisol concentrations since they provide a more stable measure, but they might not exempt of cosmetic hair treatments bias [22]. Even though some studies performed in animals and humans showed that hair cortisol concentrations increase in stress situations [22,31], the results in our caregiver population disagree.

The main limitation of our study is the small size of sample enrolled in each group which limits the power of the statistical analysis and the probability of finding wider range of biomarker levels. The parameters extraction and analysis of the physiological data is currently ongoing and further results will be published.

In summary, the value of self-perceived stress is significantly greater in the caregivers' community summited to a chronic situation of stress due to the care of an oncologic patient. The work in process may provide the baseline information to initiate specific social actions addressed to increase the 
empowerment of the caregivers' community. Our results ought to be consolidated in prospective cohort studies with larger groups of caregivers to help to perform medical actions directed to prevent comorbidities and social actions.

\section{ACKNOWLEDGMENTS}

Research supported by MINECO (FIS-PI12/00514 and TIN2014-53567-R) and by the Centro de Investigación Biomédica en Red sobre Bioingeniería, Biomateriales y Nanomedicina (CIBER-BBN) at the Instituto de Salud Carlos III de España.

\section{REFERENCES}

[1] Schulz R, Sherwood PR. Physical and mental health effects of family caregiving. Am J Nurs. 2008;108(9 Suppl):23-7; quiz 27. doi: 10.1097/01.NAJ.0000336406.45248.4c.

[2] Vitaliano PP, Zhang J, Scanlan JM. Is caregiving hazardous to one's physical health? A meta-analysis. Psychol Bull. 2003;129:946-72.

[3] Pinquart $M$, Sörensen S. Associations of stressors and uplifts of caregiving with caregiver burden and depressive mood: a meta-analysis. J Gerontol B Psychol Sci Soc Sci. 2003;58:P112-28.

[4] Oliva-Moreno J, Trapero-Bertran M, Peña-Longobardo LM, Del Pozo-Rubio R. The Valuation of Informal Care in Cost-of-Illness Studies: A Systematic Review. Pharmacoeconomics. 2017;35:331-345.

[5] Stenberg U, Ruland CM, Miaskowski C. Review of the literature on the effects of caring for a patient with cancer. Psychooncology. 2010;19:1013-25.

[6] Carmeli E. The invisibles: unpaid caregivers of the elderly. Front Public Health. 2014;2:91. doi: 10.3389/fpubh.2014.00091.

[7] Rosengren A, Hawken S, Ounpuu S, Sliwa K, Zubaid M, Almahmeed WA, et al. Association of psychosocial risk factors with risk of acute myocardial infarction in 11119 cases and 13648 controls from 52 countries (the INTERHEART study): case-control study. Lancet. 2004;364:953-62.

[8] von Känel R, Mausbach BT, Dimsdale JE, Mills PJ, Patterson TL, Ancoli-Israel S, et al. Cardiometabolic effects in caregivers of nursing home placement and death of their spouse with Alzheimer's disease. J Am Geriatr Soc. 2011;59:2037-44.

[9] Roepke SK, Allison M, Von Känel R, Mausbach BT, Chattillion EA, Harmell AL, et al. Relationship between chronic stress and carotid intima-media thickness (IMT) in elderly Alzheimer's disease caregivers. Stress. 2012;15:121-9.

[10] Siddiqui A, Madhu SV, Sharma SB, Desai NG. Endocrine stress responses and risk of type 2 diabetes mellitus. Stress. 2015;18:498-506. 
Esther García, Sira Aguiló, Adriana Arzaa, Jorge M. Garzón-Reya, Jordi Aguiló. Psychological and Physiological Profiles in Oncology Caregivers: a Multivariable Cross-Sectional Study. Transactions on Machine Learning and Artificial Intelligence, Vol 5 No4 August (2017); p: 547-557

[11] Selye H. Stress and the general adaptation syndrome. Br Med J. 1950; 1: 1383-92.

[12] Choi J, Ahmed B, Gutiérrez-Osuna R. Development and evaluation of an ambulatory stress monitor based on wearable sensors. IEEE Trans Inf Technol Biomed 2012; 16: 279-86.

[13] Thayer JF, Åhs F, Fredrikson M, Sollers JJ, Wager TD. A metaanalysis of heart rate variability and neuroimaging studies: implications for heart rate variability as a marker of stress and health. Neurosci Biobehav Rev 2012; 36: 747-56.

[14] Visnovcova Z, Mestanik M, Javorka M, Mokra D, Gala M, Jurko A, et al. Complexity and time asymmetry of heart rate variability are altered in acute mental stress. Physiol Meas 2014; 35: 1319-34.

[15] Vlemincx E, Taelman J, De Peuter S, Van Diest I, Van den Bergh O. Sigh rate and respiratory variability during mental load and sustained attention. Psychophysiology 2011; 48: 117-20.

[16] Allen AP, Kennedy PJ, Cryan JF, Dinan TG, Clarke G. Biological and psychological markers of stress in humans: focus on the Trier Social Stress Test. Neurosci Biobehav Rev 2014; 38: 94-124.

[17] De Santos-Sierra A, Ávila CS, Casanova JG, Del Pozo GB. Real-time stress detection by means of physiological signals. Recent Application in Biometrics 2011; 58: 4857-65.

[18] Haufe S, Kim JW, Kim IH, Sonnleitner A, Schrauf M, Curio G, et al. Electrophysiology-based detection of emergency braking intention in real-world driving. J Neural Eng 2014; 11: 056011.

[19] Vinkers CH, Penning R, Hellhammer J, Verster JC, Klaessens JH, Olivier B, et al. The effect of stress on core and peripheral body temperature in humans. Stress 2013; 16: 520-30.

[20] Schulz, P., Kirschbaum, C., Prüßner, J. and Hellhammer, D. Increased free cortisol secretion after awakening in chronically stressed individuals due to work overload. Stress Med.1998;14:91-97.

[21] Hjortskov, N., Garde, A. H., Ørbæk, P. and Hansen, Å. M. Evaluation of salivary cortisol as a biomarker of self-reported mental stress in field studies. Stress and Health, 2004;20: 91-98. doi:10.1002/smi.1000

[22] Russell E1, Koren G, Rieder M, Van Uum S. Hair cortisol as a biological marker of chronic stress: current status, future directions and unanswered questions. Psychoneuroendocrinology. 2012; 37(5):589-601.

[23] Yalta K, Yalta T, Sivri N, Yetkin E. Copeptin and cardiovascular disease: a review of a novel neurohormone. Int J Cardiol 2013; 167: 1750-9.

[24] Urwyler SA, Schuetz P, Sailer C, Christ-Crain M. Copeptin as a stress marker prior and after a written examination-the CoEXAM study. Stress 2015; 18: 134-7.

[25] Chrousos GP, Gold PW. The concepts of stress and stress system disorders. Overview of physical and behavioral homeostasis. JAMA. 1992; 267(9):1244-52.

[26] Cohen S, Kamarck T, Mermelstein R. A global measure of perceived stress. J Health Soc Behav 1983; 24: 385-96. 
Transactions on Machine Learning and Artificial Intelligence Vol 5 No 4, Aug 2017

[27] Monk TH. A visual analogue scale technique to measure global vigor and aff ect. Psychiatry Res 1989; 27: 89-99.

[28] Spielberger CD, Gorsuch RL, Lushene R, Vagg PR, Jacobs GA. Manual for the State-Trait Anxiety Inventory. Palo Alto, CA: Consulting Psychologists Press; 1983.

[29] Whalen KJ, Buchholz SW. The reliability, validity and feasibility of tools used to screen for caregiver burden: a systematic review. JBI Libr Syst Rev. 2009;7:1373-1430.

[30] Aguiló J, Ferrer-Salvans P, García-Rozo A, Armario A, Corbí Á, Cambra FJ, et al. Project ES3: attempting to quantify and measure the level of stress. Rev Neurol. 2015;61:405-15.

[31] Scorrano F, Carrasco J, Pastor-Ciurana J, Belda X, Rami-Bastante A, Bacci ML, et al. Validation of the longterm assessment fhypothalamic-pituitary-adrenal activity in rats using hair orticosterone as a biomarker. FASEB J. 2015;29:859-67. 